\title{
The Complex Time WKB Approximation And Particle Production
}

\author{
S.Biswas ${ }^{* a), b)}$, B.Modak ${ }^{a)}$ and A.Shaw ${ }^{* * a)}$ \\ a) Dept. of Physics, University of Kalyani, West Bengal, \\ India, Pin.-741235 \\ b) IUCAA, Post bag 4, Ganeshkhind, Pune 411 007, India \\ * email: sbiswas@klyuniv.ernet.in \\ ** email:amita@klyuniv.ernet.in \\ ()
}

\begin{abstract}
The complex time WKB (CWKB) approximation has been an effective technique to understand particle production in curved as well as in flat spacetime. Earlier we obtained the standard results on particle production in time dependent gauge in various curved spacetime. In the present work we generalize the technique of CWKB to the equivalent problems in space dependent gauge. Using CWKB, we first obtain the gauge invariant result for particle production in Minkowski spacetime in strong electric field. We then carry out particle production in de-Sitter spacetime in space dependent gauge and obtain the same result that we obtained earlier in time dependent gauge. The results obtained for de-Sitter spacetime has a obvious extension to particle production in black hole spacetime. It is found that the origin of Planckian spectrum is due to repeated reflections between the turning points. As mentioned earlier, it is now explicitly shown that particle production is accompanied by rotation of currents.
\end{abstract}




\section{INTRODUCTION}

The use of complex trajectories within the framework of Complex time WKB approximation (CWKB) has been a recent trend [1 6] to understand particle production in curved spacetime as well as in Minkowski spacetime and can be extended to understand particle production from black holes. The method is also effective in quantum cosmology especially in evaluating the wavefunction of the universe [], and to understand the preheating and reheating mechanism in inflationary cosmology [8,9]. Balian and Bloch [10] formulated an approach to quantum mechanics starting from classical trajectories with complex coordinates. In the approach, within the framework of WKB approximation wave optics is generalized to complex trajectories to build up the wave. Such a method accounts for contributions of $\exp (-c / \hbar)$ to the usual WKB wave and is found to reproduce quantitatively all quantum mechanical effects even in cases where the scattering potential varies rapidly over a distance of wavelength or less.

All complex trajectories are not allowed to contribute and one has to find a prescription for good (or, allowable) paths to be retained and how to combine them. It is question of topology in complex spaces, the topology of Stokes lines in one dimensional problems. In higher dimension one has to consider the topology of saddles, their position and height. The reader may consult Fedoryuk 11] and Knoll and Schaeffer [12 for various aspects of Stokes lines, saddles and transition matrices within the framework of WKB approximation. The ref. [12] concentrates mainly on the heavy ion scattering, calculating reflection coefficient (identified as scattering amplitude) using the roles played by Stokes lines and saddles.

We generalized the technique of [12] for complex time considering Schroedinger like equation not in space but in time and find surprising and remarkable results when applied to various expanding spacetimes. It is well known fact that in Schroedinger like equation in time (i.e., the potential is also time dependent), the vacuum state at $t \rightarrow-\infty$ and at $t \rightarrow+\infty$ are not the same and is the root cause that affects particle production.

To understand particle production in such a situation, consider a potential $V(t)$ represented as a blob from which a pair is created; both particle and anti-particle of positive energy are moving forward in time away from the blob. Using Feynmann-Stuckleberg prescription, we identify negative energy particle propagating backward in time as being equivalent to positive energy antiparticle solution moving forward in time. Thus reflecting the direction of electron coming out of the blob, we interpret the pair production as reflection of positron off the potential $V(t)$.

The particle production in flat spacetime in an uniform electric field with $A_{\mu}=(0, E t, 0,0)$ has been treated in [5] using CWKB and find the standard result. The more familiar potential $A^{\prime}{ }_{\mu}=(-E x, 0,0,0)$ is related to $A_{\mu}$ by a gauge transformation $A^{\prime}{ }_{\mu}=A_{\mu}+\partial_{\mu} \Lambda$ with $\Lambda=-E x t$. The result of particle production should be independent of the gauge i.e., should be the same for the choice $A_{\mu}$ or $A_{\mu}^{\prime}$. In the space dependent gauge (i.e., for the choice $A^{\prime}{ }_{\mu}$ ), the vacuum of the field remains the same for all time and hence no particle production can take place. To recover the standard result in space dependent gauge, we use CWKB with a tunneling interpretation and obtain the gauge independent result. The method of complex paths, enunciated by Landau in [13], has been recently used in [14] to calculate the transmission and reflection coefficients for the equivalent quantum mechanical problem. Our result for reflection and transmission coefficients in CWKB differs from [14 but the expression for Bogolubov co-efficients and vacuum persistence probability coincide with that of gauge invariant method proposed by Schwinger. In (14), the Bogolubov coefficients $\alpha$ and $\beta$ are determined from a heuristic interpretation of unitarity relation $|R|^{2}+|T|^{2}=1$ in terms of Bogolubov coefficients that differs in space dependent and time dependent gauges. However in CWKB, we employ a different procedure [3, 1, 15. and the Bogolubov coefficients are obtained in a gauge invariant way. The advantage of CWKB is that we do not require a knowledge of mode solutions and the analytic continuation $(x, t) \rightarrow-\infty$ to $(x, t) \rightarrow+\infty$ as has been done in other works [14,16]. We also do not need a prior knowledge of the gauge invariant results.

Being encouraged by the success of CWKB in space dependent gauge we next consider the deSitter spacetime and modify the previous work in the light of CWKB. We obtain the standard results usual to blackhole spacetime. The details will be placed elsewhere. We mention the main 
results obtained only for de-Sitter spacetime.

The basic problem in CWKB is to find the behaviour of a wave in a given region under the constraint of boundary conditions in other regions. During evolution, one encounters regions in which the particle (i.e., wavepacket) moves in a region of complex domain that is classically inaccessible and is characterized by turning points or saddles depending on the dimensions; but quantum mechanically there is a probability of transition in the classically unallowed region with a heuristic interpretation of tunneling. The heuristic interpretation gives a steady state of particle production. The similar situation exists in blackhole problems but here the turning points and saddles are replaced by horizons. The existence of horizon creates a separation in spacetime into portions very much akin to the behaviour of turning points such that straight trajectories become curved so as to approach or recede from the horizon exponentially slowly as seen by an external observer. A good review of black hole quantum physics is given in [16]. Unfortunately, it requires a mastery in quantum physics as well as in classical relativity to understand the conceptual issues raised by quantum mechanics in the presence of horizon.

The present paper is a critical review of particle production in curved spacetime and is a generalization of our previous works [1 5] with an extension to blackhole problems. This is done especially to understand the blackhole evaporation and to settle some of the concepts introduced in [16], that we find different in CWKB. The CWKB has the advantage to be applicable in the same way to spinor particle production and has been carried out in [3 [4.6]. The particle production in a time dependent potential is understood as follows. A purely positive frequency wave with amplitude $T$ in the infinite past $t \rightarrow-\infty$ evolves into a combination of positive and negative frequency waves in the infinite future $t \rightarrow \infty$ with negative frequency waves having an amplitude $R$ and positive frequency waves with amplitude unity. The process is viewed as a reflection in time as mentioned earlier. During discussion on spinor particle production [3 5] it was advocated that the pair production takes place due to rotation of currents from $-|J|$ to $+|J|$ as $t$ evolves from $-\infty$ to $+\infty$ and the mixing of positive frequency and negative frequency states occurs in the region between the turning points. The particle (wavepacket) moves then in complex $t$-plane. We verified this conclusion by carrying out numerical calculations in various expanding spacetimes [17, 18] with some interesting conclusions.

Many results obtained in the present work are new. The organization of the paper is follows. In section 2 we discuss the basics of CWKB applicable both in the time dependent and space dependent gauge. In section 3 we discuss the CWKB particle production in Minkowski spacetime in an uniform electric field. In section 4 . we clarify the heuristic interpretation of particle production through rotation of currents and from charge conservation and obtain the gauge invariant result even in CWKB. In section 5 we consider de Sitter spacetime in time dependent gauge and obtain the gauge invariant result. This example has an obvious extension to blackhole spacetimes.

\section{BASICS OF CWKB}

The details of complex time WKB approximation have been done in our previous works [1.99. We mention here briefly for a follow up of the text of the paper. Let us consider a one dimensional Schroedinger equation

$$
\frac{\partial^{2}}{\partial \eta^{2}}+\omega^{2}(\eta) \psi=0 .
$$

The variable $\eta$ may correspond to space or time-like variable. In CWKB we consider $\eta$ to be a complex variable and assume that $\omega(\eta)$ has complex turning points or $\omega(\eta)$ is complex between the two turning points (may be real). The turning points are given by

$$
\omega^{2}\left(\eta_{1,2}\right)=0 .
$$

We consider the case for two turning points. The method can also be generalized for higher number of turning points. Defining 


$$
S\left(\eta_{f}, \eta_{i}\right)=\int_{\eta_{i}}^{\eta_{f}} \omega(\eta) d \eta
$$

the solution of (1) in CWKB is written as

$$
\psi(\eta) \rightarrow \exp \left[i S\left(\eta, \eta_{0}\right)\right]-i R \exp \left[-i S\left(\eta, e t a_{0}\right)\right], \quad \eta \rightarrow \infty
$$

Here $\eta_{0}$ and $\eta$ are real where $\eta_{0}$ is arbitrary and $\eta_{0}>\eta$. We have neglected the WKB prefactor for simplicity. In (4) $R$ is given by

$$
R=\frac{\exp \left[2 i S\left(\eta_{1}, \eta_{0}\right)\right]}{1+\exp \left[2 i S\left(\eta_{1}, \eta_{2}\right)\right]}
$$

The derivation of (5) from global analysis in complex $\eta$-plane will be found in Knoll and Schaeffer [12]. We give here a transparent and intuitive derivation. Let a wave (to be identified as positron) starts from large and positive $\eta_{0}$, and moves leftward to reach a point $\eta$ such that $\eta<\eta_{0}$. This is represented by the first term in (4). The second term is the reflected part contribution. A wave starts at $\eta=\eta_{0}$, moving leftward reaches the turning point $\eta_{1}$ and bounces back from $\eta_{1}$ rightward to reach $\eta_{0}$. It is represented as

$$
(-i) \exp \left[i S\left(\eta_{1}, \eta_{0}\right)-i S\left(\eta, \eta_{1}\right)\right]
$$

Here $(-i)$ factor is introduced due to reflection. The contribution (6) is then multiplied by repeated reflection between the turning points $\eta_{1}$ and $\eta_{2}$ and is written as

$$
\sum_{\mu=0}^{\infty}\left[-i \exp \left\{i S\left(\eta_{1}, \eta_{2}\right)\right\}^{2 \mu}\right]=\frac{1}{1+\exp \left[2 i S\left(\eta_{1}, \eta_{2}\right)\right.} \text {. }
$$

The combined contributions (6) and (7) now comprise the second term in (4). We have used $i S\left(\eta, \eta_{1}\right)=i S\left(\eta, \eta_{0}\right)-i S\left(\eta_{1}, \eta_{0}\right)$ using relation (3). The boundary condition taken is, at $\eta \rightarrow-\infty$

$$
\psi(\eta) \rightarrow T e^{i S\left(\eta, \eta_{0}\right)}, \quad \eta \rightarrow-\infty
$$

The interpretation of (4) and (8) now runs as follows. If we consider $\exp \left[i S\left(\eta, \eta_{0}\right)\right]$ as the antiparticle solution, (4) is interpreted as the reflection of a anti-particle from the turning point $\eta_{1}$ and the reflected part is interpreted as particle moving forward in time. Here $R$ and $T$ refer to reflection and transmission coefficient. The essence of (4) and (8) is that there is no particle at $\eta \rightarrow-\infty$, but at $\eta \rightarrow+\infty$, (8) evolves into (4) and is identified as a process of pair production where the pair production amplitude $\equiv$ reflection amplitude. It is interesting to note that the similarity of (4) with the ubiquitous moving mirror example 19] and the origin of thermal spectrum. Considering $\eta$ as a time-like variable, we have been able to show using (4), (5) and (8) that in deSitter spacetime the particle creation occurs due to Hartle-Hawking vacuum decay, we also calculated the vacuum instability and found $2 \operatorname{Im} L_{\text {eff }}=\frac{4 H^{4}}{\pi^{2}} \exp \left(\frac{-m}{T_{H}}\right)$ where $T_{H}=\frac{H}{2 \pi}$ is the Hawking-deSitter temperature. The approach given here can also be generalized to problems when $\eta$ is a space like variable and the potential contains a space dependent part. We take a standard example of particle production in an uniform electric field in a space dependent gauge.

\section{CWKB PARTICLE PRODUCTION IN AN UNIFORM ELECTRIC FIELD}

In presence of an e.m. field the minimally coupled scalar field $\phi$ propagating in flat spacetime satisfies the Klein-Gordon equation

$$
\left[\left(\partial_{\mu}+i q A_{\mu}\right)\left(\partial^{\mu}+i q A^{\mu}\right)+m^{2}\right] \Phi=0 .
$$

Let us take $A^{\mu}=\left(-E_{0} x, 0,0,0\right)$. The electric field is $\vec{E}=E_{0} \hat{x}$. We write 


$$
\Phi=e^{-i \omega t} e^{i k_{y} y+i k_{z} z} \phi(x)
$$

Substituting (10) in (9) we find

$$
\frac{\partial^{2} \phi}{\partial x^{2}}+\left[\left(\omega+q E_{0} x\right)^{2}-M^{2}\right] \phi=0
$$

In (11) we have taken

$$
\begin{aligned}
& k_{\perp}{ }^{2}=k_{y}^{2}+k_{z}^{2}, \\
& M^{2}=k_{\perp}^{2}+m^{2} .
\end{aligned}
$$

The equation (11) is of the form (1) with the turning points given by

$$
\left(\omega+q E_{0} x\right)= \pm M .
$$

It is better to have an convenient form of (11) and (13) making the following change of variables

$$
\rho=\sqrt{q E_{0}} x+\frac{\omega}{q \sqrt{E_{0}}}, \lambda=\frac{M^{2}}{q E_{0}},
$$

in (11). We get

$$
\frac{\partial^{2} \phi}{\partial x^{2}}+\left(\rho^{2}-\lambda\right) \phi=0
$$

so that the turning points are at $\rho= \pm \sqrt{\lambda}$. Before we apply CWKB to (15), let us try to understand the origin of complex paths in the formalism. Let us restrict to $(1+1)$ dimension and identify $x_{c}=-\frac{\omega}{q E_{0}}$, and the acceleration $a=\frac{q E_{0}}{M}$ (now $M=m$ since $k_{\perp}^{2}=0$ ). The classical trajectory is now given by

$$
\left(x-x_{c}\right)^{2}-\left(t-t_{c}\right)^{2}=a^{-2}
$$

whereas the equation that determines the turning point reads

$$
\left(x-x_{c}+a^{-1}\right)\left(x-x_{c}-a^{-1}\right)=0,
$$

with $x_{ \pm}=x_{c} \pm a^{-1}$ being the two turning points. Considers a value $x$ between the two turning points i.e., $x_{c}-a^{-1}<x<x_{c}+a^{-1}$. We see from (17) that

$$
\left(x-x_{c}\right)^{2}-a^{-2}<0 .
$$

Using (16), we now find

$$
\left(t-t_{c}\right)^{2}<0
$$

i.e., $t$ becomes complex. This again implies from (16) that $\left(x-x_{c}\right)^{2}<0$ provided the electric field is large enough, a necessary condition of pair production. Since the potential in (11) is space dependent we have to consider the motion in $x$ plane like a scattering problem in quantum mechanics. The conditions (4) and (8) are now replaced by

$$
\psi(x) \rightarrow e^{-i S\left(-x, x_{0}\right)}-i R e^{+i S\left(-x, x_{0}\right)} \quad x \rightarrow-\infty .
$$

As $x \rightarrow+\infty,(20)$ evolves into

$$
\psi(x) \rightarrow T e^{-i S\left(x, x_{0}\right)},
$$

where $R$ is now given by 


$$
R=\frac{e^{-2 i S\left(x_{1}, x_{0}\right)}}{1+e^{-2 i S\left(+x_{1},+x_{2}\right)}} .
$$

In (21) and (22), $R$ and $T$ are respectively the reflection and transmission coefficients. We have now taken

$$
x_{1}=-\sqrt{\lambda}, x_{2}=+\sqrt{\lambda}
$$

In (21) and (22), $x_{0}$ is arbitrary. Its effect is to multiply (21) and (22) by a factor $\exp \left[i \delta\left(x_{0}\right)\right]$ where $\delta\left(x_{0}\right)$ is real, so that $|R|^{2}$ and $|T|^{2}$ do not depend on $x_{0}$. So we neglect its contribution in evaluating $|R|$ and $|T|$. However, in present example we retain it to show our claim. Going from $x$ to $\rho$ variable we now have

$$
S\left(\rho_{f}, \rho_{i}\right)=\int_{\rho_{i}}^{\rho_{f}}\left(\rho^{2}-\lambda\right)^{1 / 2} d \rho .
$$

Remembering the fact that between the turning points, $\left(\rho^{2}-\lambda\right)^{1 / 2}$ is complex, we write

$$
S\left(\rho_{f}, \rho_{i}\right)=+i \int_{\rho_{i}}^{\rho_{f}}\left(\lambda-\rho^{2}\right)^{1 / 2} d \rho .
$$

Taking $\rho_{1}=-\sqrt{\lambda}$ and $\rho_{2}=+\sqrt{\lambda}$, we find

$$
\begin{aligned}
& S\left(\rho_{1}, \rho_{0}\right)=-\frac{i \lambda \pi}{4} \\
& S\left(\rho_{1}, \rho_{2}\right)=\frac{-i \lambda \pi}{2} .
\end{aligned}
$$

Hence

$$
R=\frac{e^{i \delta\left(\rho_{0}\right)} e^{\frac{-\pi\left(k_{\perp}^{2}+m^{2}\right)}{2 q E_{0}}}}{1+e^{\frac{-\pi\left(k_{\perp}^{2}+m^{2}\right)}{q E_{0}}}}
$$

where $\delta\left(\rho_{0}\right)$ is real. The expression (27) differs from the work of Brout et. al [15] and [14]. It is therefore necessary to work out the interpretation of (21), (22) and (27) in the light of CWKB and obtain the standard Schwinger Gauge invariant result [20].

\section{CHARGE CONSERVATION AND ROTATION OF CURRENTS}

Looking back to (21) and (22) we find for $\Phi$ given in (10) [suppressing $\exp \left(i k_{y} y+i k_{z} z\right)$ factor] as

$$
\left.\begin{array}{c}
\Phi_{i} \\
\Phi_{R} \\
\Phi_{T}
\end{array}\right\}=e^{-i \omega t}\left\{\begin{array}{cl}
e^{-i S(+x)}, & x \rightarrow-\infty \\
R e^{+i S(+x)}, & x \rightarrow-\infty \\
T e^{-i S(+x)}, & x \rightarrow+\infty
\end{array}\right.
$$

Writing $S(x)$ for large argument we get

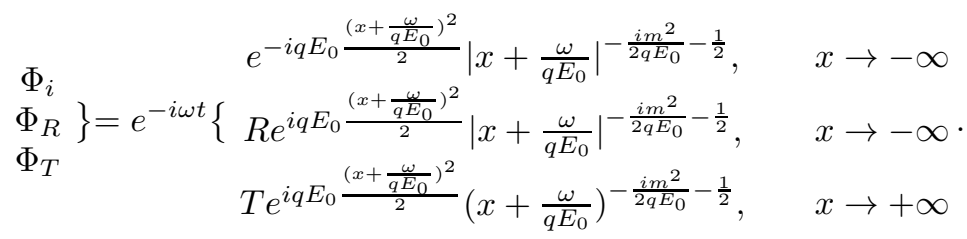


To obtain the prescription in $t$-space where $\phi_{i}, R, T$ live, we differentiate the total phase in (29) with respect to $\omega$ and set $\frac{\partial S(\text { total })}{\partial \omega}=0$. For $\phi_{i}$ we get

$$
\frac{\partial S(\text { total })}{\partial \omega}=-\left(x+\frac{\omega}{q E_{0}}\right)-t+\frac{1}{E\left|\left(x+\frac{\omega}{q E_{0}}\right)\right|}=0 .
$$

From (30) we see that as $x \rightarrow-\infty, t$ goes to $+\infty$. In a similar way, we obtain the $t$-behaviour of $\phi_{\mathrm{R}}$ and $\phi_{\mathrm{T}}$. Thus we find

$$
\begin{aligned}
\psi_{i} & \equiv I(x \rightarrow-\infty, t \rightarrow+\infty) \\
\psi_{R} & \equiv R(x \rightarrow-\infty, t \rightarrow-\infty) \\
\psi_{T} & \equiv T(x \rightarrow+\infty, t \rightarrow+\infty) .
\end{aligned}
$$

This behaviour is also obtained in 15 and is required to obtain the charge prescription in different regions. Thus $\psi_{i}$ corresponds to motion to the left at late times where $R$ describes the motion to the right at early times and $T$ to the right but at late times. Looking at (31) we note that at $t \rightarrow+\infty$ we have wave moving forward and another wave moving backward in time and is identical in prescription discussing pair production through time dependent gauge. It establishes also the heuristic interpretation of particle-antiparticle rotation through tunneling mechanism.

Let us now look at the charge assignment of various branches. For the purpose we construct the charge density using $(29)$ and neglect $\exp \left(i \overrightarrow{k_{\perp}} \cdot \overrightarrow{r_{\perp}}\right)$ term for convenience in interpretation. We get

$$
\begin{aligned}
J^{t} & =\phi_{\omega}^{*} i D_{t} \phi_{\omega}=\phi_{\omega}^{*}\left(i \overleftrightarrow{\partial_{t}}+2 q E_{0} x\right) \phi_{\omega} \\
& =2\left(\omega+q E_{0} x\right) \chi_{\mathrm{WKB}}^{*} \chi_{\mathrm{WKB}}
\end{aligned}
$$

where $\chi_{\mathrm{WKB}}$ is given in (29). At the turning point $\omega+q E_{0} x=-M, J^{t}$ is negative. Thus both $\psi_{i}$ and $\psi_{R}$ that live at negative $x$ have negative charge. This is also exemplified by (32) when $x \rightarrow-\infty$. Similarly at $\omega+q E_{0} x=+M$, we get $J^{t}$ positive i.e., $\psi_{\mathrm{T}}$, that live at $x \rightarrow+\infty$ has positive charge. In otherwords, the current $J^{t}$ undergoes a rotation from negative to positive values as it evolves from $x \rightarrow-\infty$ to $x \rightarrow+\infty$. This rotation occurs due to mixing of positive and negative frequency solutions within the turning points as envisioned earlier. Now invoking the charge conservation

$$
\left.\int d^{3} x J^{t}\right|_{t=-\infty}=\left.\int d^{3} x J^{t}\right|_{t=+\infty}
$$

we get

$$
\begin{aligned}
-|R|^{2} & =+|T|^{2}-1 \\
\text { or, }|\mathrm{R}|^{2}+|\mathrm{T}|^{2} & =+1 .
\end{aligned}
$$

In deriving (34) we have noted the fact $\psi_{i}$ and $\psi_{\mathrm{T}}$ live at $t=+\infty$, whereas $\psi_{\mathrm{R}}$ lives at $t=-\infty$. Thus we see that the unitarity relation itself is a statement of charge conservation. Thus we observe from (31) that, though we worked with a space dependent gauge, we arrive at a situation i.e., a pair at $t \rightarrow+\infty$ and no particle at $t \rightarrow-\infty$ and which is identified as pair production via (4) and (8). Thus we arrive at the same interpretation in both the gauges. To put (34) in terms Bogolubov co-efficients $\alpha$ and $\beta$, Brout et. al [15] identify

$$
|\alpha|^{2}=\frac{1}{|R|^{2}}, \quad|\beta|^{2}=\frac{|T|^{2}}{|R|^{2}}
$$

so that $|\alpha|^{2}-|\beta|^{2}=1$. However our result for $|R|^{2}$ and $|T|^{2}$ differs from $[15$ and Padmanabhan 114 and naturally the Bogolubov co-efficients will be different and would not get the Schwinger result if we follow (35). In our view (35) is not a correct choice or is an approximate relation. We know that Bogolubov co-efficients find an interpretation only through 


$$
\text { (number of pairs created in a mode } \mathrm{k} \text { ) } \equiv\left|\beta_{\mathrm{k}}\right|^{2}
$$

This is the basic definition and interpretation of $\left|\beta_{k}\right|^{2}$. To establish the correctness of CWKB, we now turn to the calculation of vacuum persistence probability to investigate the correctness of our approach or feasibility of the expression (35). It needs a calculation of $\alpha_{k}$ and $\beta_{k}$.

In CWKB, we calculate $\beta_{\omega}$ and $\alpha_{\omega}$ through a different approach. The reflection co-efficient $|R|^{2}$ is identified as the probability for the creation of one pair with wave number $\vec{k}(\equiv k l m)$. We write it as $[3,19]$

$$
|R|^{2}=\left|A_{0}\right|^{2} \omega_{k}
$$

where $A_{o}$ is the amplitude for no particle production and $\omega_{k}$ is the relative probability of creating a pair in a given mode. The probability of producing $n$ pairs with wavenumber $\vec{k}$ is

$$
P_{n}(k)=\left|A_{0}\right|^{2} \omega_{k}^{n} \text {. }
$$

The conservation of probability

$$
\sum P_{n}(k)=1
$$

then gives

$$
\left|A_{0}\right|^{2}=1-\omega_{k}
$$

Thus

$$
|R|^{2}=\left(1-\omega_{k}\right) \omega_{k} .
$$

Using the expression of $|R|^{2}$ from equation (27) in (41) we find

$$
\begin{gathered}
\omega_{\mathrm{K}}=\frac{\exp \frac{-\pi\left(k_{\perp}^{2}+m^{2}\right)}{q E_{0}}}{1+e^{\frac{-\pi\left(k_{\perp}^{2}+m^{2}\right)}{q E_{0}}}}, \\
1-\omega_{\mathrm{K}}=\frac{1}{1+e^{\frac{-\pi\left(k_{\perp}^{2}+m^{2}\right)}{q E_{0}}}} .
\end{gathered}
$$

The average number of pairs with wavenumber $\vec{k}$ is

$$
N(k)=\sum_{n=0}^{\infty} n P_{n}(k)=\frac{\omega_{\mathrm{K}}}{1-\omega_{\mathrm{K}}}
$$

Using (42) and (43) in (44) we find

$$
\begin{aligned}
N(k) & =\exp \left(\frac{-\pi\left(k_{\perp}^{2}+m^{2}\right)}{q E_{0}}\right) \\
& \equiv\left|\beta_{k}\right|^{2} \quad \text { (by definition). }
\end{aligned}
$$

This result of $\left|\beta_{\mathrm{K}}\right|^{2}$ is standard and give also the correct expression of vacuum persistence probability. The probability that the vacuum remains the vacuum is given by

$$
\begin{aligned}
\mid<0, \text { out } \mid 0, \text { in }>\left.\right|^{2} & =\prod_{k}\left|A_{0}\right|^{2}, \\
& =\prod_{k}\left(1-\omega_{\mathrm{K}}\right), \\
& =\prod_{k} \frac{1}{1+\left|\beta_{k}\right|^{2}}, \quad(\operatorname{using}(43) \text { and }(45)), \\
& =\exp \left(-\sum_{k} \ln \left(1+\left|\beta_{k}\right|^{2}\right)\right)
\end{aligned}
$$


The equation (46) delivers the probability to find no pairs at future times. From (44) and (45), we see that though our expression for $|R|$ differs from [15], the results for Bogolubov co-efficient and the vacuum persistence probability turn out to be the same as the standard result of Schwinger [20]. In our approach

$$
\begin{aligned}
& \left|\beta_{k}\right|^{2}=e^{\left(\frac{-\pi\left(k_{\perp}^{2}+m^{2}\right)}{q E}\right)} \\
& \left|\alpha_{k}\right|^{2}=1+e^{\left(\frac{-\pi\left(k_{\perp}^{2}+m^{2}\right)}{q E_{0}}\right)} .
\end{aligned}
$$

The results (44) - (47) suggest that the identification of Bogolubov co-efficients from the unitarity or charge conservation relation $|R|^{2}+|T|^{2}=1$ is not a judicious choice. Replacing the summation in (46) by integration, we get exactly as in 15

$$
\mid<0, \text { out } \mid 0, \text { in }>\left.\right|^{2}=\exp \left(\frac{-E L T}{2 \pi} \ln \left(1+e^{\frac{-m^{2} \pi}{E}}\right)\right) \text {, }
$$

in $(1+1)$ dimension and

$$
\mid<0, \text { out } \mid 0, \text { in }>\left.\right|^{2}=\frac{-E^{2} T V}{4 \pi^{2}} \ln \left(1+e^{\frac{-m^{2} \pi}{E}}\right),
$$

in $(1+3)$ dimension. We understand that Padmanabhan 21] has obtained related results in this context and will be publishing them seperately.

Now the essence of the calculation establishes that

i) the complex paths taken in CWKB are all allowable and good paths and takes into account all the quantum mechanical contributions within the framework of semiclassical approximation and ii)the interpretation of pair production in CWKB is a gauge independent result.

The equality of (46) and (45) with the Schwinger result raises a question. In [15] as well as in [14], one also obtains the same results using (35), though having the same answer for Bogolubov coefficients and vacuum persistence probability. The difference occurs due to analytic continuation. When one goes from $\psi_{\mathrm{T}}$ at $x \rightarrow+\infty$ to $\psi_{\mathrm{I}}+R \psi_{\mathrm{R}}$ at $x \rightarrow-\infty$, not all the complex paths are taken into account in (14) and (15), whereas the CWKB does it correctly. We like to place the details in future; however similar view will also be found in [22].

\section{PARTICLE PRODUCTION IN DE-SITTER SPACETIME}

In standard black hole spacetime, of which de-Sitter is an example, particle production was discovered by Hawking [23] using semi-classical analysis. In his method, the semi-classical propagator of a scalar field propagating in Schwarzchild spacetime is analytically continued in the time variable t to complex values. Using time dependent gauge in de-Sitter spacetime we obtained [3] that the appropriate vacuum for an inflationary early universe is the Hartle-Hawking vacuum and this vacuum acts as a blackbody of temperature $T_{H}=H / 2 \pi$, the standard result. It is therefore necessary to obtain the same result in space dependent gauge for a de-Sitter spacetime.

Consider a spacetime

$$
d s^{2}=B(r) d t^{2}-B^{-1}(r) d r^{2}-r^{2}\left(d \theta^{2}+\sin ^{2} \theta\right) d \phi^{2},
$$

where $B(r)=1-H^{2} r^{2}$, in de-Sitter spacetime. For massless Klein-Gordon equation we have

$$
\square \phi=0 .
$$

In the spacetime (50), with

$$
\phi=\psi(r, t) Y_{l}^{m}(\theta, \phi)
$$


the equation (51) reads

$$
\frac{1}{B(r)} \frac{\partial^{2} \psi}{\partial t^{2}}-\frac{1}{r^{2}} \frac{\partial}{\partial r}\left(r^{2} B(r) \frac{\partial \psi}{\partial r}\right)+\frac{l(l+1)}{r^{2}} \psi=0 .
$$

Making the ansatz $\psi=\exp (-i \omega t) f_{\omega}$ in $(53)$ we get

$$
\frac{B(r)}{r^{2}} \frac{\partial}{\partial r}\left(r^{2} B(r) \frac{\partial f_{\omega}}{\partial r}\right)+\left(\omega^{2}-\frac{l(l+1)}{r^{2}} B(r)\right) f_{\omega}=0 .
$$

Introducing $d r^{*}=\frac{d r}{r^{2} B(r)}$ in (54), we find

$$
\frac{\partial^{2} f_{\omega}}{\partial r^{* 2}}+r^{4}\left(\omega^{2}-\frac{l(l+1)}{r^{2}} B(r)\right) f_{\omega}=0 .
$$

This is like our one dimensional Schroedinger equation in previous section with

$$
S(r)=\int^{r}+r^{2}\left(\omega^{2}-\frac{l(l+1)}{r^{2}}\right)^{1 / 2} \frac{d r}{r^{2} B(r)} .
$$

For $B(r)=1-H^{2} r^{2}$, the horizon is at $R=H^{-1}$ and the turning points are at $r= \pm \infty$. The $r \rightarrow-\infty$ has to be identified at the left of the horizon. The method of CWKB is now applied with the turning point at $r_{1}=+X,-X$, where $X$ is sufficiently large. As the particle enters the horizon radius, it moves in complex $(t, r)$-plane and we apply CWKB in the following way. we now write as before

$$
f_{\omega}(x) \underset{x \rightarrow-\infty}{\rightarrow} e^{-2 i S(-x,-A)}+R e^{-2 i S(-x,-A)},
$$

where

$$
R=-\frac{i e^{-2 i S(-X,-A)}}{1+e^{-2 i S(X,-X)}} .
$$

Starting from $-A>-X$, as we proceed towards the right at the other turning point $+X$ we have to cross the horizon. Therefore reaching near horizon we move in upper half complex plane in a semi-circular path and back on the real axis. Thus

$$
S(X,-A)=\int_{-A}^{-\varepsilon}+\int_{S . C}+\int_{\varepsilon}^{X} .
$$

Here $\mathrm{A}$ is an arbitrary point.

In (59), we take $l=0$ as it gives the most contribution (see ref. 15]) so that

$$
S\left(x_{1}, X_{2}\right)=\int_{x_{1}}^{x_{2}} \frac{\omega}{B(r)} d r .
$$

As is evident from (58), the contribution from $A$, i.e.,

$$
S(A)=\int^{A} \frac{\omega}{B(r)} d r
$$

multiplies (58) as $\exp [i S(A)]$ and hence we do not take its contribution in (59) for calculating $R$. We evaluate (59) and find

$$
\int_{-A}^{X} \equiv-\frac{i \pi \omega}{2 H}+\delta_{1}, \int_{-X}^{X} \equiv-\frac{i \pi \omega}{H}+\delta_{2}, \quad \int_{S . C}=0,
$$

where $\delta_{1}$ and $\delta_{2}$ are real. Hence

$$
|R|=\frac{\exp \left(\frac{-\pi \omega}{H}\right)}{1+\exp \left(\frac{-2 \pi \omega}{H}\right)} .
$$

Thus we recover the Planckian spectrum with $T=\frac{H}{2 \pi}$. This result is remarkable. Thus we arrive at gauge invariant result even in CWKB. 


\section{DISCUSSION}

Using CWKB we have obtained the gauge invariant result even in curved spacetime similar to electromagnetic example. It has been argued that [14] the tunneling picture given in the present work fails in curved spacetime and in blackhole spacetime. However in the present work we judiciously circumvent the claim and obtained the Hawking result very nicely. We mention here the important results and would shortly explore the details.

It is worthwhile to mention some comparison with the standard works. Suppose we neglect the repeated reflection between the turning points and determine the Bogolubov coefficients from (41). We find, neglecting the denominator in (27)

$$
|R|^{2}=\exp (-\pi M)
$$

where

$$
M=\frac{\sqrt{k_{\perp}^{2}+m^{2}}}{q E_{0}}
$$

We now calculate $\omega_{k}$ from (41). We find

$$
\begin{gathered}
\omega_{k}=\frac{1}{2}(1 \pm \sqrt{1-4 \exp (-\pi M)}) \\
1-\omega_{k}=\frac{1}{2}(1 \mp \sqrt{1-4 \exp (-\pi M)})
\end{gathered}
$$

Taking the negative sign before the square root for $\omega_{k}$, we get from (44) and (45)

$$
\left|\beta_{k}\right|^{2} \simeq \frac{\exp (-\pi M)}{1-\exp (-\pi M)}
$$

provided $\exp (-\pi M) \ll 1$.

Hence

$$
\left|\alpha_{k}\right|^{2}=1+\left|\beta_{k}\right|^{2}=\frac{1}{1-\exp (-\pi M)}
$$

so that

$$
\left|\beta_{k}\right|^{2}=\left|\alpha_{k}\right|^{2} \exp (-\pi M)
$$

Carrying out this calculation for de-Sitter and Schwarzchild spacetime, we find similar results. For de-Sitter spacetime, we get $T=\frac{H}{2 \pi}$ and for Schwarzchild we get $T=\frac{1}{8 \pi M}$ in $(1+3)$ dimensions.

This exercise also exemplifies the usefulness of CWKB that takes more quantum corrections than the other methods and gives a partial answer to the analytic continuation, $x \rightarrow-\infty$ to $x \rightarrow+\infty$, ensuring that the method of CWKB is more accurate and transparent, from the standpoint of physical arguments, than the other methods.

[1] S.Biswas, J.Guha and N.G.Sarkar, Pramana J. Phys. 40467 (1993)

[2] S.Biswas, J.Guha and N.G.Sarkar, Pramana J. Phys. 42319 (1994)

[3] S.Biswas, J.Guha and N.G.Sarkar, Class. Quantum Grav. 121591 (1995) 
[4] J.Guha, D.Biswas, N.G.Sarkar and S.Biswas, Class. Quantum Grav. 121641 (1995)

[5] S.Biswas, J.Guha and P.Dasgupta, IJMPA 10319 (1994)

[6] N.G.Sarkar and S.Biswas, Pramana J. Phys. 50109 (1998)

[7] S.Biswas, B.Modak and D.Biswas, Phys. Rev. D55 4673 (1996)

[8] N.G.Sarkar, Ph. D. Thesis: A study on some aspects of particle production, 61-63 (1998)

[9] A.Shaw, D.Biswas, B.Modak and S.Biswas, 'Particle Production, Back Reaction and Singularity Avoidance', (To be published in Pramana)

[10] R.Balian and C.Bloch, Ann. Phys. (N.Y.) 85514 (1974)

[11] M.V.Fedoryuk, Asymptotic analysis (Springer-Verlag, Berlin) (1993)

[12] J.Knoll and R.Schaeffer, Ann. Phys. (N.Y.), 97307 (1976)

[13] L.D.Landau and E.M.Lifshitz, Quantum mechanics (Non-relativistic theory, Course of Theoretical Physics, Volume 2), Pergamon Press, N.Y. (1975)

[14] K.Srinivasan and T.Padmanabhan, Facets of tunneling: particle production in external fields, InterUniversity Centre for Astronomy and Astrophysics Preprint (1998)

[15] A.O.Barut and I.H.Duru, ICTP/89/179, 'Pair production in an electric field in a time dependent gauge (1979)

[16] R.Brout, S.Masser, R.Parentani and Ph.Spindel, Physics Report 260329 (1995)

[17] N.G.Sarkar and S.Biswas, 'Particle production in de-Sitter spacetime', IJMPA (in press) (1999)

[18] N.G.Sarkar and S.Biswas, 'Particle production in expanding spacetime', Pramana J. Phys., (communicated) (1998)

[19] N.D.Birrel and P.C.W.Davies, 'Quantum field in curved space', Cambridge University Press), Cambridge (1982)

[20] J.Schwinger, Phys. Rev. 82, 'On Gauge invariance and vacuum Polarization, 664 (1951)

[21] T.Padmanabhan, Private Communication (1998)

[22] N.B.Narozhny and A.I.Nikishov, Issues in Intense-field quantum electrodynamics, (Proceedings of the Lebedev Physics Institute of the Academy of Science of the USSR, Vol. 168), ed. Ginzburg, V.L. 235-236 (1987)

[23] J.B.Hartle and S.W.Hawking, Phys. Rev. D189 13 (1976)

\section{ACKNOWLEDGMENT}

A.Shaw acknowledges the financial support from ICSC World Laboratory, LAUSSANE during the course of the work. 